\title{
A case of bortezomib-associated thrombotic microangiopathy in a multiple myeloma patient
}

Journal of Onco-Nephrology

2020, Vol. 4(I-2) 46-5I

(C) The Author(s) 2019

Article reuse guidelines:

sagepub.com/journals-permissions DOI: $10.1177 / 2399369319894343$

journals.sagepub.com/home/jnp

(S)AGE

\author{
Nuno Moreira Fonseca' (iD), Filipa Cardoso', \\ Manuel Monteiro2, Mário Góis ${ }^{1,3}$, Helena Sousa ${ }^{1,3}$, \\ Teresa Fidalgo ${ }^{4}$ iD, Joaquim Calado ${ }^{1,5}$ \\ and Fernando Nolasco ${ }^{\prime}$
}

\begin{abstract}
Bortezomib is a first-generation proteasome inhibitor used in the treatment of multiple myeloma. We present a case of a 70-year-old woman with multiple myeloma, who presented thrombotic microangiopathy with multi-organ involvement thrombotic microangiopathy (ocular, cardiac, and renal) after bortezomib initiation. A kidney biopsy confirmed the diagnosis of thrombotic microangiopathy. A temporal relation between bortezomib exposure and thrombotic microangiopathy onset was seen in the absence of other concurrent medication or disease known to cause thrombotic microangiopathy, and thrombotic microangiopathy was only resolved after drug discontinuation. The exact pathophysiological mechanism remains unknown. To our knowledge, this is the second biopsy-proven published case of bortezomib-associated thrombotic microangiopathy. Since bortezomib is extensively used for treating patients with multiple myeloma, prescribing clinicians should maintain a high index of suspicion of this potentially fatal complication.
\end{abstract}

\section{Keywords}

Bortezomib, thrombotic microangiopathy, multiple myeloma, nephrotoxicity

Date received: 26 August 2019; accepted: 15 November 2019

\section{Introduction}

Thrombotic microangiopathy (TMA) is a serious, often fatal, medical condition characterized by microvascular thrombotic occlusion. TMA consists of a spectrum of multiple syndromes, including thrombotic thrombocytopenic purpura (TTP), hemolytic uremic syndrome (HUS), atypical HUS, and drug-induced TMA (DITMA). Bortezomib (BTZ), a proteasome inhibitor, is extensively used for treating patients with multiple myeloma. TMA has been described in multiple myeloma (MM) patients who received BTZ. In this report, we describe a case of a biopsy-proven TMA in an MM patient after BTZ exposure.

\section{Case report}

We report a case of a Caucasian woman with a past medical history of hypertension, depression, and breast cancer diagnosed 10 years before, treated with breast-conserving surgery, chemotherapy (5-fluorouracil, epirubicin, cyclophosphamide), radiotherapy, and hormonal therapy.

In 2017, at 70 years of age, our patient was diagnosed with stage 3 (international staging system), IgG kappa MM

\footnotetext{
'Nephrology Department, Centro Hospitalar Universitário de Lisboa Central, Lisbon, Portugal

${ }^{2}$ Internal Medicine Department, Centro Hospitalar e Universitário de Lisboa Central, Lisbon, Portugal

${ }^{3}$ Laboratory of Renal Morphology, Centro Hospitalar e Universitário de Lisboa Central, Lisbon, Portugal

${ }^{4}$ Clinical Hematology Department, Centro Hospitalar e Universitário de Coimbra, Coimbra, Portugal

${ }^{5}$ ToxOmics, Centre for Toxicogenomics and Human Health, Nova

Medical School, Lisbon, Portugal

Corresponding author:

Nuno Moreira Fonseca, Nephrology Department, Centro Hospitalar Universitário de Lisboa Central, Rua da Beneficiência 8, 1069-166 Lisbon, Portugal.

Email: nuno.mf@nyu.edu
} 


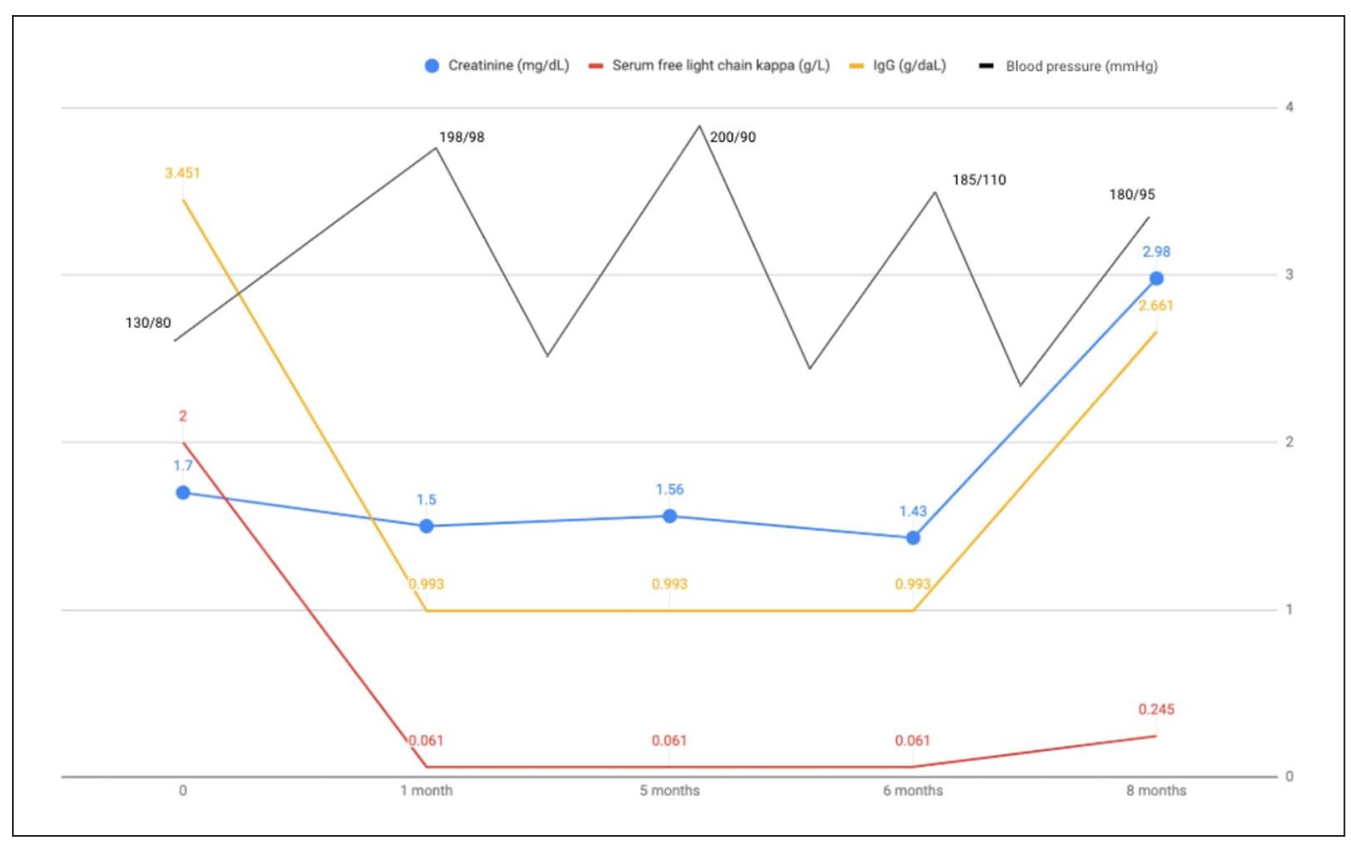

Graph I. Patient clinical and analytical evolution.

after routine follow-up blood work at another institution, 9 months before admission in our hospital. At the time of diagnosis, the patient was found to have IgG in the level of $34.51 \mathrm{~g} / \mathrm{L}$, serum-free Kappa light chains in the level of $2000 \mathrm{mg} / \mathrm{L}$, a bone marrow biopsy showing 65\% plasma cells with a monoclonal kappa population, and a skull $\mathrm{X}$-ray presenting bone lesions. Renal function (creatinine $1.7 \mathrm{mg} / \mathrm{dL})$ had deteriorated from baseline $(0.5 \mathrm{mg} / \mathrm{dL})$, and hypercalcemia was not present (Graph 1).

The patient was started on VMP chemotherapy regimen (BTZ $1.3 \mathrm{mg} / \mathrm{m}^{2}$ subcutaneous on days $1,4,8$, and 11 ; Melphalan $9 \mathrm{mg} / \mathrm{m}^{2}$, orally on days $1,2,3$, and 4 ; and Prednisone $60 \mathrm{mg} / \mathrm{m}^{2}$ orally on days $1,2,3$, and 4 of a 21 -day cycle) as an outpatient. She was admitted to another hospital 1 day after the third dose of the first cycle of BTZ with complaints of weakness and decreased visual acuity. On admission, she presented with severe hypertension, and fundoscopy showed papilledema with flame-shaped retinal hemorrhages. Blood work revealed anemia (hemoglobin $(\mathrm{Hb}) 6.0 \mathrm{~g} / \mathrm{dL}$ ) and thrombocytopenia (platelets $35,000 / \mu \mathrm{L}$ ), with no change in baseline serum creatinine. Results of coagulation tests were normal. Head magnetic resonance imaging (MRI) showed acute infarct on the right caudate head, and T2/FLAIR hyperintensity of subcortical white matter in the anterior and upper frontal region bilaterally. A lumbar puncture was performed, excluding intracranial hypertension. The patient received transfusions of blood red cells and platelets. After stabilization of blood pressure control and improvement of visual complaints, the patient was discharged.

Chemotherapy was continued in ambulatory. One week after the end of the fourth VMP cycle, the patient was admitted to another hospital for acute onset dyspnea in the setting of hypertensive pulmonary edema. CT-angiography of the chest showed bilateral pleural effusion, pericardial effusion, and excluded pulmonary embolism. Electrocardiogram and biological markers of myocardial necrosis were unremarkable. Kidney function was unchanged (serum creatinine of $1.56 \mathrm{mg} / \mathrm{dL}$ ), and the patient did not present thrombocytopenia or aggravated anemia. The patient was discharged after 6 days. One month later, the patient was admitted to the same hospital for a similar episode of hypertensive pulmonary edema. Both episodes were assumed to be due to acute cardiac insufficiency.

After discharge, the patient continued VMP therapy as an outpatient. While on the sixth cycle of VMP, the patient was admitted for the third time for hypertensive pulmonary edema in the setting of cardiac insufficiency. The patient presented Coombs-negative hemolytic anemia (Hb $6.9 \mathrm{~g} / \mathrm{dL}$, lactate dehydrogenase (LDH) $433 \mathrm{U} / \mathrm{L}$, decreased haptoglobin, and elevated indirect bilirubin), thrombocytopenia (platelets $60,000 / \mu \mathrm{L}$ ), and worsened kidney function (serum creatinine of $2.98 \mathrm{mg} / \mathrm{dL}$ ). The patient received red blood cell transfusions. Renal ultrasound sound showed normal-sized kidneys without signs of obstruction. During hospitalization, renal function progressively deteriorated over the following days reaching a serum creatinine of $4.5 \mathrm{mg} / \mathrm{dL}$. Additional work-up showed an increase in both in IgG level (47.83 vs $26.61 \mathrm{~g} / \mathrm{L} 1 \mathrm{month}$ before) and free Kappa light chains level (245 vs $70 \mathrm{mg} / \mathrm{L} 1 \mathrm{month}$ before), and kidney failure was attributed to multiple myeloma progression. At this point, BTZ was assumed to be associated with the patient's episodes of cardiac decompensation, and myeloma treatment was changed to cyclophosphamide and 


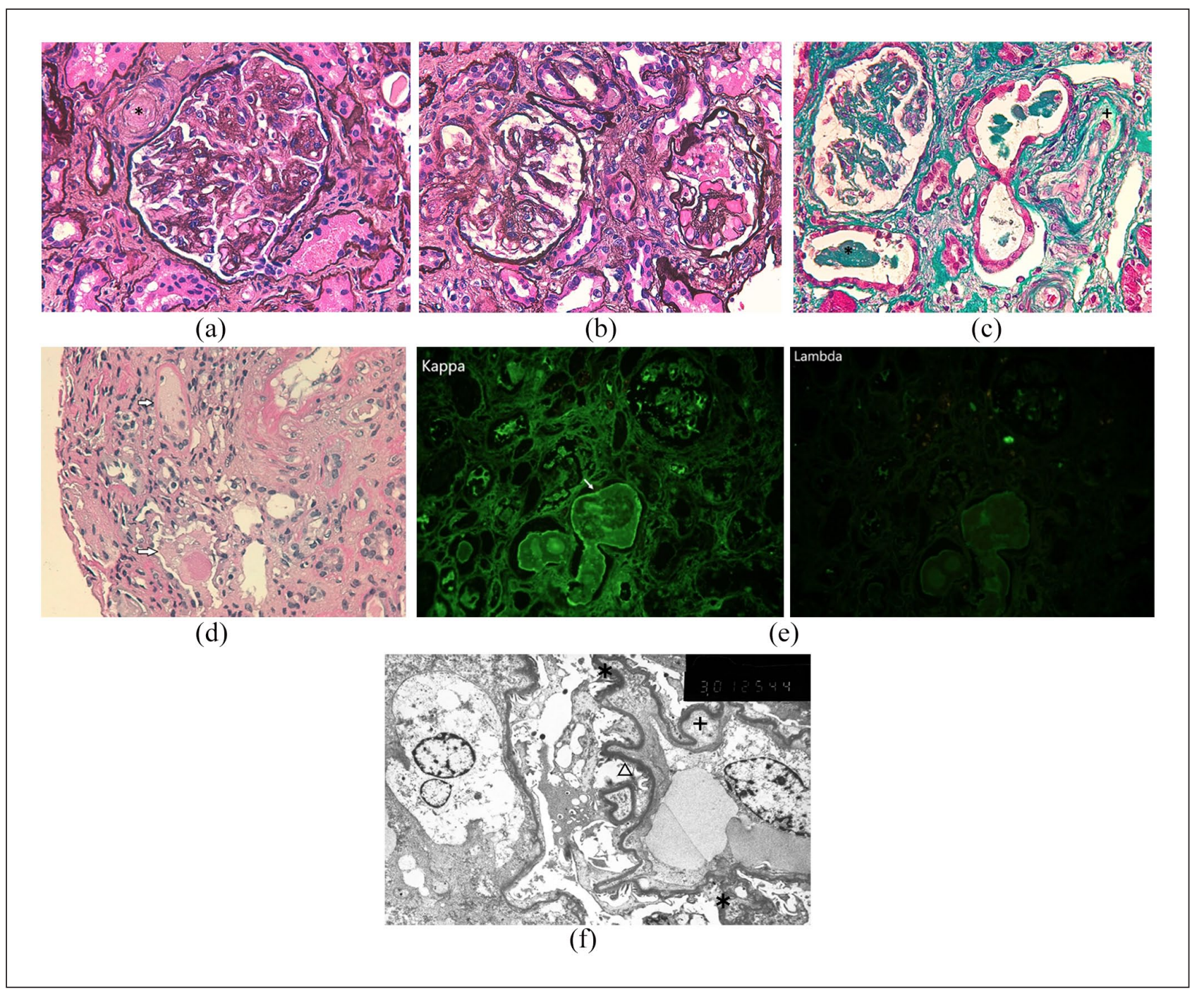

Figure I. Kidney biopsy showing TMA. (A, B, C) Light Microscopy shows mesangiolysis, focal double contours and a thrombus in the afferent arteriole $\left(^{*}\right)$ (A - Jones methenamine silver, 400x); Intraglomerular thrombus (B - Jones methenamine silver, 400x). Interstitial fibrosis is present throughout the cortical region, intimal mucoid edema is present in arterioles $(+)(C-$ Masson's thricrome, 400x). Distal tubules are filled with myeloma casts with inflammatory reaction (arrow) (D -Periodic Acid Schiff, 400x). Immunofluorescence was positive for kappa cylinders, shown by white arrow, and negative for lambda. ( $\mathrm{E}$ - paraffin embedded tissue, 400x). Electron microscopy shows glomerular basement membrane wrinkling (*), expanded subendothelial space $(+)$ and podocyte foot process effacement $(\triangle)$. ( $F$ - paraffin embedded tissue).

prednisolone. Renal function further deteriorated with ensuing anuria, prompting the decision to initiate hemodialysis. At this time, she was transferred to our nephrology department, where she maintained hemolytic anemia and thrombocytopenia requiring frequent red blood cell transfusions. Activity of ADAMTS13 was not significantly decreased $(0.30 \mathrm{UI} / \mathrm{mL})$, and inhibiting autoantibodies of ADAMTS13 were not detected.

A renal biopsy was performed and showed both thrombotic microangiopathy and kappa cast nephropathy and excluded other conditions associated with monoclonal gammopathies like membranoproliferative glomerulonephritis, light chain proximal tubulopathy, monoclonal light chain deposition disease, and cryocrystalglobulinemia. The kidney sample contained eight glomeruli, all ischemic, with areas of mesangiolysis and some with focal double contours. Thrombi were observed in three afferent arterioles (Figure 1(a)). Some glomeruli presented thrombus in the capillaries (Figure 1(b)) and some arterioles presented intimal mucoid edema (Figure 1(c)). Myeloma casts with inflammatory reaction were detected in distal tubules (Figure 1(d)). Immunofluorescence on formalin-fixed paraffin-embedded tissue was positive for kappa light chain and negative for lambda (Figure 1(e)). Electron microscopy, performed on formalin-fixed paraffin-embedded tissue, showed preservation artifacts and glomerular basement 
membrane wrinkling, fibrin thrombus, expanded subendothelial space, focal endothelial cell injury, and podocyte foot process effacement. (Figure 1(f)).

In addition to kappa light chain cast nephropathy, we established the diagnosis of BTZ-associated TMA. The patient was given additional red blood cell transfusions while further platelet transfusions were held. She remained dialysis-dependent and was transferred to another institution to resume myeloma treatment with daratumumab.

\section{Discussion}

This patient represents the ninth case of TMA associated with BTZ, which has been reported in the literature, and the second case which is biopsy-proven. Cases of TMA associated with monoclonal gammopathy have also been described. ${ }^{1}$ Our patient's progressing MM may have contributed to TMA development along with BTZ administration, since drug administration was time-related to TMA onset.

One day after the third dose of BTZ, the patient developed acute hemolytic anemia with malignant hypertension and papilledema. These findings are commonly present in TMA. Additional presence of abnormalities of visual perception with MRI findings localized to the subcortical white matter could constitute an episode of reversible posterior leukoencephalopathy syndrome (RPLS). RPLS is an increasingly recognized clinical radiographic syndrome of heterogeneous etiologies, commonly associated with hypertensive disorders, renal disease, and TMA, being more frequent in women. Both malignant hypertension and endothelial dysfunction are known to contribute to the development of this transitory syndrome. ${ }^{2}$

Acute heart failure motivated the subsequent hospitalizations. While it is commonly believed that TMA rarely presents with symptomatic cardiac involvement, acute heart failure is a potentially fatal complication of thrombotic microangiopathies with an estimated incidence of $9.5 \%$ in one of the largest published institutional series. ${ }^{3}$ Several mechanisms have been proposed including highoutput heart failure secondary to anemia and myocardial ischemia secondary to microvascular thrombi. ${ }^{4}$ Although we were not able to rule out obstructive coronary artery disease with cardiac catheterization, the patient had a normal EKG and showed no elevation of biological markers of myocardial necrosis.

Finally, progressive renal failure was thought to be consistent with multiple myeloma progression. Albeit an absolute increase in the serum M-component $>5 \mathrm{~g} / \mathrm{L}$, kidney biopsy revealed histological findings of TMA in addition to cast nephropathy.

There are numerous causes of TMA. Attributing a complication to a specific drug requires the fulfillment of multiple criteria including a table pathophysiological pathway, a chronological association, and the absence of concurrent disease or medication associated with the same complication.

In this case, there was a clear chronological relation between TMA and BTZ administration. Hypertension, fragmentation hemolysis, and thrombocytopenia presented soon after treatment initiation. Further drug cycles induced subsequent hospital admissions for ocular, cardiac, and renal TMA manifestations. In addition, the hematology findings of TMA were only resolved after drug discontinuation.

Current medication did not include other drugs known to cause TMA such as type 1 interferon, gemcitabine, bevacizumab, sunitinib, cyclosporine, or tacrolimus. ${ }^{5,6}$

In regard to concurrent disease, reduced activity of ADAMTS13 or autoantibodies against it were absent, as well as anti-factor-H antibodies. Although stool was not analyzed, the patient did not present signs or symptoms of Shiga toxin producing Escherichia coli O157 infection, namely, diarrhea or fever. Genetic analysis was unremarkable for mutations in the genes coding for proteins regulating the alternative complement pathways (atypical HUS (aHUS)), that included search for allelic variants by next-generation sequencing in the following genes: $C F H$, CD46 (MCP), CFI, C3, THBD, CFB, CFHR5, CFHRI CFHR3, CFHR4, and DGKE. In addition, no large rearrangements were found in the CFH-CFHR locus by multiplex ligand probe amplification. The patient did not present any rheumatologic disorder or active malignancy. TMA can also occur in the setting of hematopoietic cell transplantation (HCT) and exposure to graft-versus-host disease. ${ }^{7}$ Despite being a common treatment for multiple myeloma patients, our patient had not undergone a HCT. As previously discussed, TMA can also be associated with monoclonal gammopathy. Recently, the International Kidney and Monoclonal Gammopathy Research Group gave TMA a provisional status as one of the kidney lesions associated with monoclonal gammopathy of renal significance. Monoclonal proteins can mimic polyclonal immunoglobulins involved in pathogenesis of TTP and aHUS. ${ }^{7}$ Therefore, although we cannot entirely exclude the contribution of MM to this patient's TMA, the temporal relation to BTZ exposure as well the lack of anti-factor-H activity and the presence of normal ADAMTS13 activity make this an unlikely event.

There have been eight cases of BTZ-associated acute kidney injury (AKI) with evidence of underlying TMA, of which only one was biopsy-proven (Table 1). ${ }^{8-14}$ We report the second case of BTZ-associated TMA with both kidney biopsy showing evidence of TMA and genetic analysis excluding mutations for the regulation of the alternative complement pathway, with clinical recurrence after drug re-exposure.

DITMA is an acquired condition resulting from exposure to a drug. Pathophysiology varies according to the causative drug and may include different pathways such as immunologic reactions with formation of drug-dependent 


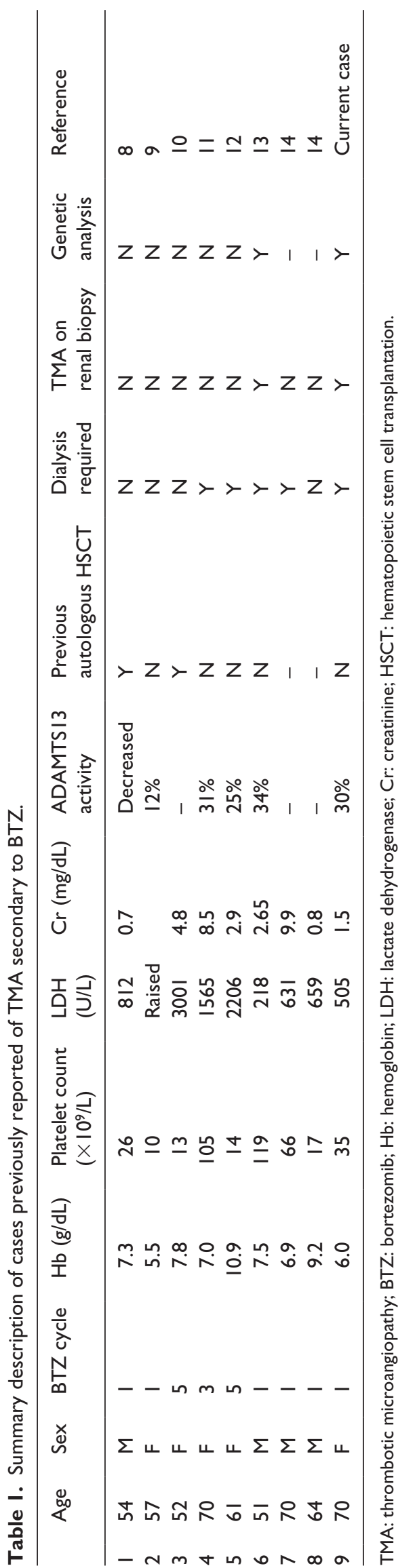

antibodies or direct tissue toxicity. The mechanisms of BTZ-induced TMA remain uncertain. BTZ action as a proteasome inhibitor suppresses vascular endothelial growth factor (VEGF) secretion, leading to a loss of the healthy fenestrated phenotype of endothelium, promoting microvascular injury and TMA. Treatment of DITMA is limited to drug avoidance and supportive care.

In summary, we describe what to our knowledge is the ninth case of BTZ-associated TMA, and second case which is biopsy-proven. This is also the second case published which is corroborated by a negative screening for complement mutations. BTZ is widely used in the treatment of MM, and prescribing clinicians should maintain a high index of suspicion of this potentially fatal complication in case of hemolytic anemia onset. The possible mechanisms remain to be clarified.

\section{Declaration of conflicting interests}

The author(s) declared the following potential conflicts of interest with respect to the research, authorship, and/or publication of this article: N.M.F. has received financial support from Astellas, AstraZeneca, Bayer, Bial, Fresenius Medical Care, Fulbright Commission, GlaxoSmithKline, Menarini, and Sanofi.

\section{Funding}

The author(s) received no financial support for the research, authorship, and/or publication of this article.

\section{ORCID iDs}

Nuno Moreira Fonseca (iD https://orcid.org/0000-0003-0821-2764

Teresa Fidalgo (iD https://orcid.org/0000-0003-4495-4431

\section{References}

1. Yui JC, Garceau D, Jhaveri KD, et al. Monoclonal gammopathy-associated thrombotic microangiopathy. $\mathrm{Am} \mathrm{J}$ Hematol 2019; 94(10): E250.

2. Fugate JE and Rabinstein AA. Posterior reversible encephalopathy syndrome: clinical and radiological manifestations, pathophysiology, and outstanding questions. Lancet Neurol 2015; 14(9): 914-925.

3. Gami AS, Hayman SR, Grande JP, et al. Incidence and prognosis of acute heart failure in the thrombotic microangiopathies. Am J Med 2005; 118(5): 544-547.

4. Vaughn JL, Moore JM and Cataland SR. Acute systolic heart failure associated with complement-mediated hemolytic uremic syndrome. Case Rep Hematol 2015; 2015: 327980.

5. Al-Nouri ZL, Reese JA, Terrell DR, et al. Drug-induced thrombotic microangiopathy: a systematic review of published reports. Blood 2015; 125(4): 616-618.

6. Saleem R, Reese JA and George JN. Drug-induced thrombotic microangiopathy: an updated systematic review, 2014-2018. Am J Hematol 2018; 93(9): E241-E243.

7. Rosenthal J. Hematopoietic cell transplantation-associated thrombotic microangiopathy: a review of pathophysiology, diagnosis, and treatment. J Blood Med 2016; 7: 181-186. 
8. Morita R, Hashino S and Shirai S. Thrombotic microangiopathy after treatment with bortezomib and dexamethasone in a patient with multiple myeloma. Int J Hematol 2008; 88(2): 248-250.

9. Moore $\mathrm{H}$ and Romeril K. Multiple myeloma presenting with a fever of unknown origin and development of thrombotic thrombocytopenic purpura post-bortezomib. Intern Med $J$ 2011; 41(4): 348-350.

10. Salmenniemi U and Remes K. Thrombotic microangiopathy associated with bortezomib treatment in a patient with relapsed multiple myeloma. Hematol Rep 2012; 4(2): e13.

11. Mehta N, Saxena A and Niesvizky R. Unexpected outcome (positive or negative) including adverse drug reactions: bortezomib-induced thrombotic thrombocytopaenic purpura. BMJ Case Rep 2012; 2012: bcr2012006461.

12. Chan KL, Filshie R, Nandurkar H, et al. Thrombotic microangiopathy complicating bortezomib-based therapy for multiple myeloma. Leuk Lymphoma 2015; 56(7): 2185-2186.

13. Van Keer J, Delforge M, Dierickx D, et al. Renal thrombotic microangiopathy associated with the use of bortezomib in a patient with multiple myeloma. Case Rep Hematol 2016; 2016: 6020691.

14. Yui JC, Van Keer J, Weiss BM, et al. Proteasome inhibitor associated thrombotic microangiopathy. Am J Hematol 2016; 91(9): E348-E352. 